\title{
Impact of chemotherapy in the prognosis of non- small-cell lung cancer patients with severe to very severe COPD
}

This article was published in the following Dove Press journal: International Journal of COPD

\section{Weigang Dong \\ Yan Du \\ Shuping Ma}

Department of Respiratory and Critical Care Medicine, Tianjin Chest Hospital, Tianjin, People's Republic of China
Correspondence: Shuping Ma

Department of Respiratory and Critical Care Medicine, Tianjin Chest Hospital,

Taierzhuang South Road No 26I, Jinnan

District, Tianjin, People's Republic

of China

Tel +86 I86 22809337

Fax +86 2288185338

Email mashuping60000@126.com
Background: The aim of the study was to investigate if first-line chemotherapy improves total survival time in non-small-cell lung cancer (NSCLC) patients complicated with severe to very severe COPD.

Materials and methods: This retrospective observational clinical study included 267 consecutive NSCLC patients with COPD complications at the Department of Respiratory and Critical Care Medicine of Tianjin Chest Hospital between January 2009 and January 2018. Sixty-nine evaluable patients were included. The clinical characteristics, toxicity profile, objective response rate, and prognosis were analyzed and compared between patients receiving and those not receiving chemotherapy.

Results: Forty-five and 24 patients received first-line chemotherapy plus supportive care and supportive care alone, respectively. Kaplan-Meier curves showed that patients receiving chemotherapy had a statistically significant 6-month longer median overall survival (OS) than that of patients receiving supportive care alone (14.0, 95\% CI: 8.5-19.5 vs 8.0, 95\% CI: 6.4-9.6, respectively) $\left(\mathrm{chi}^{2}=8.857, P=0.003\right)$. In the multivariate Cox proportional hazard model adjusted for the most relevant variables, the adjusted hazard ratio (HRadj) differed significantly for the receipt of chemotherapy (HRadj $=0.4464,95 \% \mathrm{CI}$ : $0.2495-0.7988 ; P=0.0066$ ) but not for gender (HRadj=0.8527, 95\% CI: 0.4461-1.6298; $P=0.6297$ ), age (HRadj=1.0021, 95\% CI: 0.9609-1.0451; $P=0.9214)$, histology (HRadj=1.4422, 95\% CI: 0.6959-2.9889; $P=0.3247)$, cancer stage (HRadj=1.9098, 95\% CI: $0.8607-4.2375 ; P=0.1116)$, performance status score (HRadj=1.5155, 95\% CI: 0.7523-3.0529; $P=0.2446)$, lung function (HRadj=1.3856, 95\% CI: $0.7149-2.6857 ; P=0.3341$ ), or respiratory symptoms (HRadj=1.0518, 95\% CI: $0.6032-1.8342$; $P=0.8586)$. Patients with grade $3 / 4$ adverse reactions accounted for $29 \%(13 / 45)$ of the chemotherapy group.

Conclusion: The results indicated that chemotherapy may improve the OS of NSCLC patients with severe to very severe COPD.

Keywords: survival, toxicity, first-line chemotherapy, GOLD grade

\section{Introduction}

Lung cancer is the most common type of malignant tumor, accounting for over $12.7 \%$ of cancer cases worldwide. It is estimated to become the fifth leading cause of deaths globally by $2020 .{ }^{1}$ COPD is a frequent comorbidity of lung cancer; a large proportion $(40 \%-70 \%)$ of lung cancer patients also have COPD. ${ }^{2,3}$ This comorbidity greatly increases the incidence of death. Kiri et al reported that the 3-year survival rate of cancer patients without COPD is twice that of patients complicated with COPD. ${ }^{4}$ Nonsmall-cell lung cancer (NSCLC) is the major type of cancer in these patients. 
According to the China Pulmonary Health study, a recent national cross-sectional survey, public awareness of COPD is low even though a large proportion of the population is affected. ${ }^{5}$ This may partly explain the phenomenon in People's Republic of China that most COPD patients have progressed to a severe or very severe disease stage at their first hospital visit. Patients with poorer lung function have a higher risk of developing lung cancer. ${ }^{6}$ For lung cancer sufferers complicated with severe to very severe COPD, chemotherapy rather than surgery may benefit them more. ${ }^{7}$ However, the National Comprehensive Cancer Network guideline uses performance status (PS) score rather than COPD severity as a criterion for recommending chemotherapy to lung cancer patients. ${ }^{8}$

Previous clinical studies have indicated that comorbidities such as mild to moderate COPD had no evident adverse impact on the prognosis of patients with NSCLC receiving first-line chemotherapy. ${ }^{9,10}$ However, the same may not be true for those complicated with severe to very severe COPD who present with serious cough, dyspnea, sputum, and other respiratory symptoms and who often also have cardiovascular or cerebrovascular complications. ${ }^{11-14}$ Chemotherapy may have significant hazardous effects on these patients. ${ }^{15,16}$ Therefore, it is unclear if the therapeutic benefits of chemotherapy outweigh the accompanying adverse effects.

The objective of the present study was to investigate if first-line chemotherapy improved the total survival time in NSCLC patients complicated with severe to very severe COPD.

\section{Materials and methods Study subjects}

This retrospective observational clinical study was conducted at a single site in Tianjin, People's Republic of China. The medical records of 267 consecutive NSCLC patients with COPD complications visiting the Department of Respiratory and Critical Care Medicine of Tianjin Chest Hospital between January 2009 and January 2018 were retrieved from the institutional hospital information system and were discriminatively reviewed. This study received ethical approval from the Ethics Review Committee of Tianjin Chest Hospital (Approval No. 2018LW-001). The conduct of the study was in compliance with the principles stated in the Declaration of Helsinki. The subjects' privacy and personal identity information in this study have been fully protected. All data have been anonymized. The need for individual consent was waived by the committee because of the retrospective nature of the study.
A total of 198 patient records were eliminated based on the following pre-defined exclusion criteria: 1 ) receiving chemotherapy after lung cancer surgery (16 cases); 2) unavailability of lung function test information (130 cases); 3 ) mild to moderate COPD (44 cases); and 4) unavailability of information on follow-up visits (eight cases). A total of 69 patients were eligible for inclusion. The process of patient screening and inclusion is shown in a flowchart in Figure 1.

Among the 69 patients, 45 and 24 received first-line chemotherapy together with supportive care and supportive care alone, respectively. Supportive care for NSCLC patients with COPD included anti-infectives, hemostatics, pain-killers, and nutrition support.

The diagnostic criteria for lung cancer were as described in the 2004 World Health Organization classification of lung tumors. ${ }^{17}$

COPD was diagnosed according to the spirometric criteria of the Global Initiative for Chronic Obstructive Lung Disease as follow: 1) non-COPD was defined as $\mathrm{FEV}_{1} / \mathrm{FVC} \geq 70 \%$, 2) mild COPD was defined as $\mathrm{FEV}_{1} / \mathrm{FVC}<70 \%$ and $\mathrm{FEV}_{1} \geq 80 \%$ of the predicted, 3) moderate COPD was defined as $\mathrm{FEV}_{1} / \mathrm{FVC}<70 \%$ and $50 \% \leq \mathrm{FEV}_{1}<80 \%$ of the predicted, 4) severe COPD was defined as $\mathrm{FEV}_{1} / \mathrm{FVC}$ of $<70 \%$ and $30 \% \leq \mathrm{FEV}_{1}<50 \%$ of the predicted, and 5) very severe COPD was defined as an $\mathrm{FEV}_{1} / \mathrm{FVC}<70 \%$ and $\mathrm{FEV}_{1}<30 \%{ }^{18}$

\section{Data collection}

A self-designed data collection form was used to gather information on patient age, gender, smoking history and status (pack-years), symptoms related to COPD, PS score, pulmonary function, laboratory test results, histology, cancer

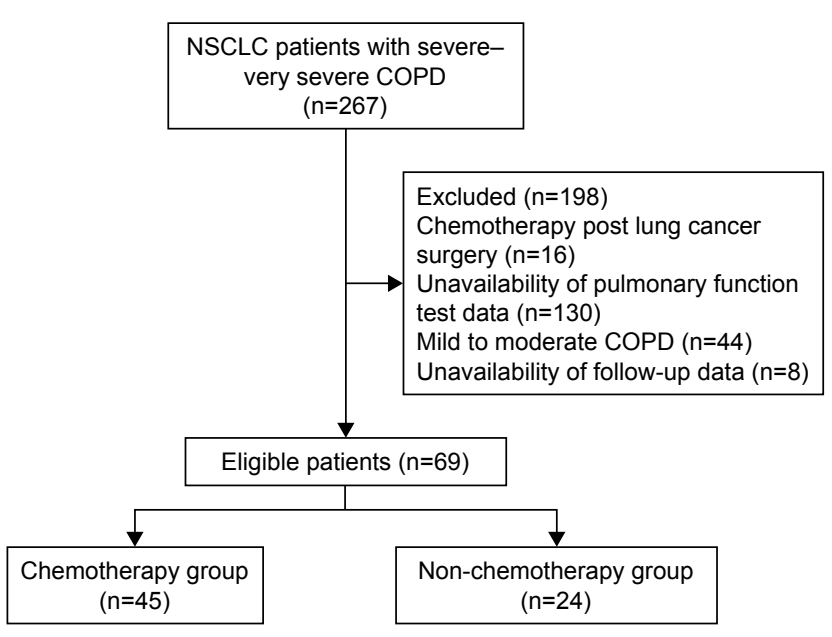

Figure I Patient screening and inclusion flow chart. Abbreviation: NSCLC, non-small-cell lung cancer. 
stage, treatment intervention, chemotherapy toxicity, and survival status, if available.

\section{Outcome and toxicity measurement}

The clinical benefits of chemotherapy were measured using the Response Evaluation Criteria in Solid Tumors. According to these criteria, the patient response was classified as complete response (CR), partial response (PR), stable disease (SD), or progressive disease (PD). Based on these measurements, composite outcomes including disease control rate $(\mathrm{DCR}, \mathrm{CR}+\mathrm{PR}+\mathrm{SD})$ and objective response rate $(\mathrm{ORR}$, $\mathrm{CR}+\mathrm{PR})$ were determined.

Overall survival (OS), defined as the time from lung cancer diagnosis to death, was measured as an endpoint outcome.

Any adverse events were graded according to the Common Terminology Criteria for Adverse Events version 4.0.

\section{Statistical analysis}

SPSS for Windows, version 16.0, was used for statistical analysis. Continuous data were described as mean and standard deviation. Student's $t$-tests were used to test differences between the chemotherapy and the non-chemotherapy groups if the original data followed a normal distribution; otherwise, Mann-Whitney $U$ tests were used.

Categorical data were expressed as proportions or incidence rates. For inter-group comparisons of most outcomes, chi-squared or Fisher's exact tests were used. To compare OS between groups, log-rank tests were used. A multivariate survival analysis using a Cox regression model was performed to investigate the impact of chemotherapy on patient survival. The "enter" method was used to select variables. $P$-values less than 0.05 indicated statistically significant differences.

\section{Results \\ Patient characteristics}

The patient characteristics and baseline data are presented in Table 1.

Compared to patients in the non-chemotherapy group, patients in the chemotherapy group were younger; however, this difference was not statistically significant. The two patient groups also differed in terms of histological subtypes. In the chemotherapy group, squamous cell carcinoma was the most dominant subtype (22/45) and cases of adenosquamous carcinoma were present. Adenocarcinoma was the most dominant subtype (12/24) in the non-chemotherapy group, and no cases of adenosquamous carcinoma were present. This difference was not statistically significant. Furthermore, in terms of cancer stage, stage III dominated in the chemotherapy group (22/45), while stage IV dominated in the non-chemotherapy group (11/24), a difference that was also not statistically different. In summary, no statistically significant variation was present between the two groups with respect to gender; smoking status; PS; COPD-relevant symptoms; pulmonary function; or laboratory data including creatinine, alanyl transferase, aspartate transaminase, r-glutamyltransferase, and albumin concentrations.

\section{Treatment and response}

All patients received first-line platinum-based doublet chemotherapy including pemetrexed in combination with platinum (nine cases), gemcitabine plus platinum (16 cases), vinorelbine plus platinum (six cases), paclitaxel plus platinum (four cases), or docetaxel plus platinum (ten cases).

Fourteen patients were treated for one or two cycles, 15 were treated for three or four cycles, and 16 were treated for five or six cycles.

After treatment, patient response was classified as PR, SD, PD, and not evaluated for five, 16, 19, and five patients, respectively. The resulting ORR and DCR in the chemotherapy group were $12.5 \%$ and $45 \%$, respectively.

\section{Prognosis}

The Kaplan-Meier curves for patients in the chemotherapy and non-chemotherapy groups are presented in Figure 2.

Four of the 45 patients in the chemotherapy group survived during our follow-up visits, whereas only one of the 24 patients in the non-chemotherapy group survived. The Kaplan-Meier curves showed that patients having received chemotherapy had a 6-month longer median OS than that of patients having only received supportive care (14.0, 95\% CI: $8.5-19.5$ in the chemotherapy group vs $8.0,95 \% \mathrm{CI}$ : 6.4-9.6 in the non-chemotherapy group), a difference that was statistically significance $\left(\mathrm{chi}^{2}=8.857, P=0.003\right)$.

Survival analysis was also performed using a multivariate Cox proportional hazard model adjusted for potentially relevant variables. The adjusted hazard ratio (HRadj) was statistically significant only for the receipt of chemotherapy (HRadj=0.4464, 95\% CI: 0.2495-0.7988; $P=0.0066$ ) and not for gender $(\mathrm{HRadj}=0.8527,95 \% \mathrm{CI}$ : 0.4461-1.6298; $P=0.6297$ ), age (HRadj=1.0021, 95\% CI: 0.9609-1.0451; $P=0.9214)$, histology $(\mathrm{HRadj}=1.4422$, 95\% CI: 0.6959 2.9889; $P=0.3247$ ), cancer stage (HRadj=1.9098, 95\% CI: 0.8607-4.2375; $P=0.1116)$, PS score $($ HRadj=1.5155, 95\% CI:0.7523-3.0529; $P=0.2446)$, lung function $(\mathrm{HRadj}=1.3856$, 95\% CI:0.7149-2.6857; $P=0.3341$ ), or respiratory symptoms 
Table I Patient characteristics and baseline physiological data

\begin{tabular}{|c|c|c|c|}
\hline & Chemotherapy group & Non-chemotherapy group & $P$-value \\
\hline$N$ & 45 & 24 & \\
\hline Male sex & 37 & 18 & 0.6919 \\
\hline Age, years & $67.89 \pm 7.18$ & $69.50 \pm 6.82$ & 0.2668 \\
\hline Smoking history, never smoker & 6 & I & 0.4339 \\
\hline Smoking status, pack-years & $46.78 \pm 26.52$ & $50.11 \pm 34.76$ & 0.854 \\
\hline Symptoms related to COPD & & & 0.8678 \\
\hline No symptom, $\mathrm{n}$ & 18 & 8 & \\
\hline Cough and sputum, $\mathrm{n}$ & 6 & 2 & \\
\hline Dyspnea, $\mathrm{n}$ & 2 & 1 & \\
\hline Cough, sputum, and dyspnea, $\mathrm{n}$ & 19 & 13 & \\
\hline PS score & & & 0.2044 \\
\hline I & 11 & 4 & \\
\hline 2 & 34 & 18 & \\
\hline 3 & 0 & 2 & \\
\hline Histology & & & 0.1134 \\
\hline Adenocarcinoma & 16 & 12 & \\
\hline Squamous cell carcinoma & 22 & 10 & \\
\hline Large cell carcinoma & $\mathrm{I}$ & 2 & \\
\hline Adenosquamous carcinoma & 6 & 0 & \\
\hline Stage & & & 0.7925 \\
\hline IA & 2 & 0 & \\
\hline IIA & 0 & 1 & \\
\hline IIB & 3 & 2 & \\
\hline IIIA & 11 & 4 & \\
\hline IIIB & 11 & 6 & \\
\hline IV & 18 & 11 & \\
\hline \multicolumn{4}{|l|}{ Lung function test } \\
\hline $\mathrm{FEV}_{1}, \mathrm{~L}$ & $1.06 \pm 0.31$ & $1.02 \pm 0.25$ & 0.8353 \\
\hline $\mathrm{FEV}_{1}, \%$ & $39.38 \pm 8.28$ & $4 I .7 I \pm 8.87$ & 0.1776 \\
\hline FVC, L & $2.20 \pm 0.58$ & $1.99 \pm 0.40$ & 0.0876 \\
\hline FVC, \% & $63.14 \pm 12.49$ & $63.80 \pm 11.76$ & 0.9598 \\
\hline GOLD grade & & & 0.8163 \\
\hline Severe, $\mathrm{n}$ & 35 & 20 & \\
\hline Very severe, $\mathrm{n}$ & 10 & 4 & \\
\hline \multicolumn{4}{|l|}{ Laboratory test } \\
\hline ALB, $g / L$ & $38.33 \pm 4.31$ & $37.13 \pm 3.59$ & 0.2468 \\
\hline AST, U/L & $20.05 \pm 16.29$ & $19.17 \pm 9.41$ & 0.5164 \\
\hline ALT, U/L & $17.97 \pm 12.84$ & $|7.32 \pm| 2.8 \mid$ & 0.6276 \\
\hline r-GT, U/L & $48.47 \pm 97.56$ & $31.29 \pm 23.44$ & 0.2867 \\
\hline $\mathrm{Cr}$, umol/L & $70.14 \pm 16.10$ & $65.15 \pm 14.92$ & 0.3225 \\
\hline
\end{tabular}

Abbreviations: ALB, albumin; ALT, alanyl transferase; AST, aspartate transaminase; Cr, creatinine; GOLD, Global Initiative for Chronic Obstructive Lung Disease; PS, performance status; r-GT, r-glutamyltransferase.

(HRadj=1.0518, 95\% CI: 0.6032-1.8342; $P=0.8586$; Table 2).

\section{Toxicity}

The administered chemotherapies were generally tolerable. Gastrointestinal reactions were the most frequently reported adverse responses, affecting 38\% (17/45) of patients, but only one $(2 \%)$ patient experienced serious (grade 3 ) gastrointestinal adverse reactions. Myelosuppression was another commonly reported adverse drug reaction. In this study, patients presenting grade 3/4 leukopenia, neutropenia, or anemia together accounted for $24 \%$ (11/45) of the chemotherapy 


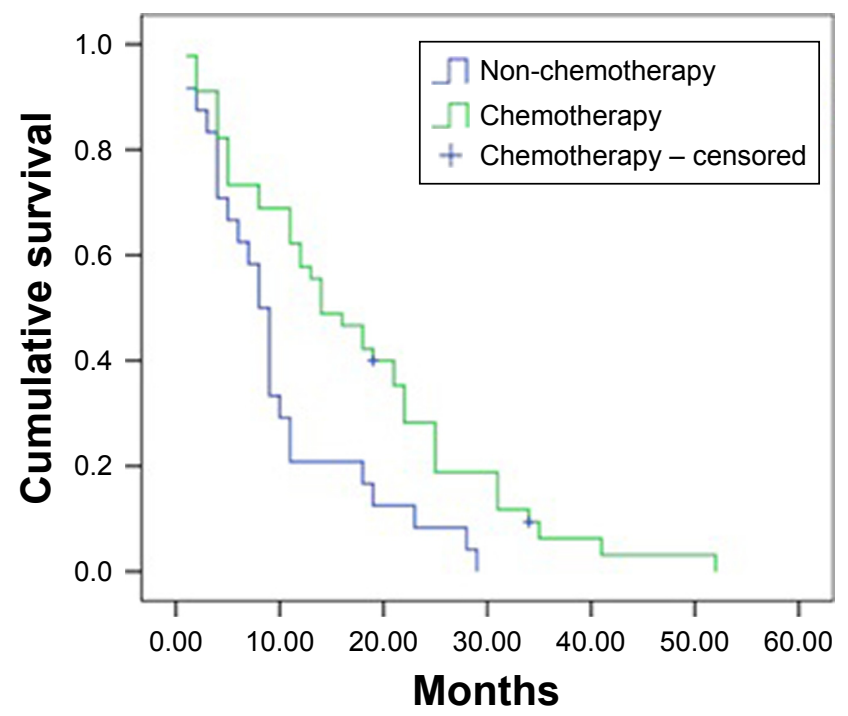

Figure 2 Kaplan-Meier curves for chemotherapy and non-chemotherapy patients.

group. The incidence rates of grade $3 / 4$ adverse reactions for leukopenia, neutropenia, anemia, and thrombocytopenia were $9 \%(4 / 45), 11 \%(5 / 45), 9 \%(4 / 45)$, and 9\% (4/45), respectively. The complete chemotherapy toxicity profiles are shown in Table 3.

\section{Discussion}

To our knowledge, this is the first clinical report on the benefit of chemotherapy in NSCLC patients with severe to very severe COPD. In the present study, the median OS was 14 months in the chemotherapy group and 8 months in the non-chemotherapy group. This finding suggests that chemotherapy prolonged the OS time of NSCLC patients with severe to very severe COPD. In other words, the severity of COPD may not be an important factor when determining whether to administer chemotherapy.

Idiopathic pulmonary fibrosis and COPD are chronic respiratory diseases characterized by evident respiratory symptoms, progressive loss of lung function, and the degeneration of functional abilities. They are also associated with increased risks of developing lung cancer. ${ }^{6,19}$ Previous studies have found chemotherapy to be beneficial for late-stage NSCLC patients complicated with idiopathic pulmonary fibrosis, with a reported median OS of 10.6-11.4 months. ${ }^{20,21}$ Similarly, reports suggest the positive effects of first-line chemotherapy on the prognosis and safety of patients with NSCLC and mild to moderate COPD. ${ }^{9}$ However, little attention has been paid to NSCLC patients with severe to very severe COPD. A 2014 study found that advanced COPD did not have a significant deleterious impact on OS following tyrosine kinase inhibitors (TKI) therapy or first-line chemotherapy. The study concluded that these therapies could benefit late-stage NSCLC patients despite their advanced COPD comorbidities. ${ }^{10}$ However, this previous study had some limitations. It included both small cell lung cancer and NSCLC patients, who have different prognoses or toxicities related to chemotherapy. Moreover, the treatment interventions involved chemotherapy and epidermal growth factor receptor-TKI therapy. These interventions may have variant response rates and different adverse reactions. ${ }^{6,22} \mathrm{By}$ contrast, the present study focused solely on NSCLC and first-line platinum-based doublet chemotherapy that yielded more accurate and reliable results.

Compared to previous prospective, randomized clinical research on late-stage NSCLC patients, the median survival time was slightly longer in our study (14 months in the present study vs $10.1-13.9$ months) ${ }^{23-25}$ There are several possible explanations. First, 64\% (29/45) of patients in the present study were categorized as having stage IIIB or IV disease, with other patients having milder stages, whereas all patients in the previous studies were stage IIIB or IV. Second, 36\% (16/45) of patients in the chemotherapy group in our study had adenocarcinoma, and more than half $(9 / 16$, $56 \%$ ) of the patients received pemetrexed plus platinum treatment. ${ }^{26}$

Table 2 Summary of the results of multivariate analysis of independent risk factors related to the prognosis of lung cancer patients

\begin{tabular}{|c|c|c|c|}
\hline & HR & $95 \% \mathrm{Cl}$ & $P$-value \\
\hline Chemotherapy & 0.4464 & $0.2495-0.7988$ & 0.0066 \\
\hline Male & 0.8527 & $0.446 \mathrm{I}-\mathrm{I} .6298$ & 0.6297 \\
\hline Age & 1.0021 & $0.9609-1.0451$ & 0.9214 \\
\hline Histology ${ }^{a}$ & 1.4422 & $0.6959-2.9889$ & 0.3247 \\
\hline Stage ${ }^{b}$ & 1.9098 & $0.8607-4.2375$ & 0.1116 \\
\hline PS score & 1.5155 & $0.7523-3.0529$ & 0.2446 \\
\hline GOLD grade ${ }^{c}$ & 1.3856 & $0.7 \mid 49-2.6857$ & $0.334 I$ \\
\hline Respiratory symptoms & 1.0518 & $0.6032-1.8342$ & 0.8586 \\
\hline
\end{tabular}

Notes: a Adenocarcinoma and squamous cell carcinoma vs adenosquamous carcinoma + large cell carcinoma. ${ }^{b}$ Stage IA + IIA + IIB vs IIIA + IIIB + IV. ' $\mathrm{Grade} 3$ vs grade 4 . Abbreviation: GOLD, Global Initiative for Chronic Obstructive Lung Disease; PS, performance status. 
Table 3 Toxicity profile of patients in the chemotherapy group (unit: cases)

\begin{tabular}{|c|c|c|c|c|c|}
\hline & Grade I & Grade 2 & Grade 3 & Grade 4 & Total \\
\hline Leukopenia & 5 & 6 & 3 & 1 & 15 \\
\hline Neutropenia & 5 & 4 & 4 & 1 & 14 \\
\hline Anemia & 7 & 3 & 4 & 0 & 14 \\
\hline Thrombocytopenia & 2 & 0 & 4 & 0 & 6 \\
\hline Gastrointestinal reaction & 12 & 4 & 1 & 0 & 17 \\
\hline Fatigue & 9 & 5 & 2 & 0 & 16 \\
\hline
\end{tabular}

Moreover, we observed lower ORR and DCR in the chemotherapy group (12.5\% and $45 \%$, respectively) than in previous studies of NSCLC patients $(38.9 \%$ and $58.3 \%$, respectively) complicated with mild to moderate COPD who also received first-line chemotherapy. ${ }^{9}$ Two reasons have been proposed to explain this difference. According to a review study, people with squamous cell carcinoma are more likely to develop tolerance to chemotherapy than those with adenocarcinoma. ${ }^{27}$ In our study, over half of the patients in the chemotherapy group had squamous cell carcinoma and may have been more likely to have a poorer outcome; however, most patients in the previous study were histologically diagnosed with adenocarcinoma. Furthermore, NSCLC patients are more likely to develop chemotherapy tolerance if they experience signs or symptoms of hypoxia. ${ }^{28}$ Therefore, it makes sense that the patients with severe to very severe COPD in our study probably had more severe hypoxia and poorer outcomes than those with mild or moderate COPD in the previous study.

Myelosuppression and gastrointestinal reactions are among the most commonly reported adverse effects of chemotherapy. Patients suffering from grade $3 / 4$ adverse reactions accounted for $29 \%$ (13/45) of the chemotherapy group and $11 \%(5 / 45)$ had neutropenia. The incidence rate of adverse reactions was lower than that (40\%) reported in a previous study. ${ }^{29}$ There are two possible explanations. First, patients in the present study received fewer treatment cycles than those in the previous study. In the present study, 31\% (14/45) of patients received one or two cycles of chemotherapy. Second, $20 \%(9 / 45)$ of patients received pemetrexed plus platinum treatment, which has a better safety profile than the other types of chemotherapy. ${ }^{26}$

Infection is a leading cause of acute exacerbation of COPD (AECOPD). ${ }^{30,31}$ Leukopenia following chemotherapy increases the risk of infection, and infections could be lifethreatening for patients with severe to very severe COPD. A previous study reported that chemotherapy did not adversely impact the safety of patients with mild to moderate COPD. ${ }^{9}$ However, the effect of severe to very severe COPD on the prognosis and safety of NSCLC patients requires further investigation.

Inhaled corticosteroid (ICS), long-acting beta-agonists (LABAs), and long-acting muscarinic antagonists (LAMAs) are standard therapeutic agents for COPD that lower the risk of the development of AECOPD, delay lung function deterioration, and improve patient quality of life. In a retrospective analysis of the medical records of 174 NSCLC plus COPD patients undergoing surgical resection, Yamanashi et al reported that the perioperative administration of ICSs had no impact on the incidence of postoperative respiratory complications. ${ }^{32}$ In a retrospective study on COPD patients who underwent surgical resection for primary lung cancer, Takegahara et al reported that preoperative management with LABAs or LAMAs not only reduced postoperative lung complications but also improved long-term survival. ${ }^{33}$ However, the possible effect of the regular use of ICS, LABA, or LAMA on chemotherapy side effects and prognosis has scarcely been discussed and requires further investigation.

Chemotherapy-related adverse reactions and drug resistance greatly affect the prognosis of lung cancer patients with COPD complications. With the advancement of radiological technology and development of therapeutic equipment, the indications for radiotherapy have gradually expanded and the adverse reactions have been better controlled. However, radiation pneumonitis may present a fatal complication for lung cancer patients with COPD, especially in those with severe to very severe COPD. It is critical to recommend radiotherapy to the right population to maximize patient benefits. Studies have investigated the relationship between the size of lung cancer masses or tumor shrinkage rate during radiotherapy and patient prognosis ${ }^{34,35}$ to identify the characteristics of the target patient population for radiotherapy. Moreover, emerging immunotherapy methods have brought new hope for lung cancer treatment. A randomized, double-blind prospective study found that among patients with stage III unresectable NSCLC who received chemoradiotherapy, those in the durvalumab group had significantly longer progression-free survival than those in the placebo 
group (16.8 vs 5.6 months) with no difference with respect to adverse events. ${ }^{36}$ The results of that study suggested that durvalumab could be a potentially effective complementary treatment for stage III NSCLC patients receiving standard treatment. Our study findings suggested that first-line chemotherapy improved the prognosis of patients with severe to very severe COPD and lung cancer. However, the role of radiotherapy and immunotherapy in this patient group requires investigation.

Our study has several limitations. First, we only investigated prognostic factors that are both important and easily accessible (including age, gender, histology, stage, PS score, treatment, and complication severity) and may have excluded other relevant factors such as patient immune, patient psychological health, and family economic statuses. Second, patients in the non-chemotherapy group may have received supportive care at hospitals other than the study site. This may cause inaccuracy in our analysis of patient prognosis due to differences in supportive care administered in different hospitals. Finally, the findings of this retrospective study require cautious interpretation, and future studies with larger sample sizes are warranted.

\section{Conclusion}

The results of the present study indicate that chemotherapy may improve the OS of NSCLC patients with severe to very severe COPD. Moreover, the COPD severity or lung function status may not be important factors for decisionmaking regarding chemotherapy; however, further studies are required for validation.

\section{Author contributions}

WD and YD contributed to study design and data collection. SM contributed to data analysis. All authors contributed to data analysis, drafting and revising the article, gave final approval of the version to be published, and agree to be accountable for all aspects of the work.

\section{Disclosure}

The authors report no conflicts of interest in this work.

\section{References}

1. Ferlay J, Shin HR, Bray F, Forman D, Mathers C, Parkin DM. Estimates of worldwide burden of cancer in 2008: GLOBOCAN 2008. Int J Cancer. 2010;127(12):2893-2917.

2. Loganathan RS, Stover DE, Shi W, Venkatraman E. Prevalence of COPD in women compared to men around the time of diagnosis of primary lung cancer. Chest. 2006;129(5):1305-1312.

3. Congleton J, Muers MF. The incidence of airflow obstruction in bronchial carcinoma, its relation to breathlessness, and response to bronchodilator therapy. Respir Med. 1995;89(4):291-296.
4. Kiri VA, Soriano J, Visick G, Fabbri L. Recent trends in lung cancer and its association with COPD: an analysis using the UK GP Research Database. Prim Care Respir J. 2010;19(1):57-61.

5. Wang C, Xu J, Yang L, et al. Prevalence and risk factors of chronic obstructive pulmonary disease in China (the China Pulmonary Health [CPH] study): a national cross-sectional study. Lancet. 2018;391(10131): 1706-1717.

6. Mannino DM, Aguayo SM, Petty TL, Redd SC. Low lung function and incident lung cancer in the United States: data From the First National Health and Nutrition Examination Survey follow-up. Arch Intern Med. 2003;163(12):1475-1480.

7. Qin J, Li G, Zhou J. Characteristics of elderly patients with COPD and newly diagnosed lung cancer, and factors associated with treatment decision. Int J Chron Obstruct Pulmon Dis. 2016;11(Issue 1): $1515-1520$.

8. Ettinger DS, Wood DE, Akerley W, et al. NCCN Guidelines Insights: Non-Small Cell Lung Cancer, Version 4. 2016. J Natl Compr Canc Netw. 2016;14(3):255-264.

9. Omote N, Hashimoto N, Morise M, et al. Impact of mild to moderate COPD on feasibility and prognosis in non-small cell lung cancer patients who received chemotherapy. Int J Chron Obstruct Pulmon Dis. 2017; 12:3541-3547

10. Izquierdo JL, Resano P, El Hachem A, Graziani D, Almonacid C, Sánchez IM. Impact of COPD in patients with lung cancer and advanced disease treated with chemotherapy and/or tyrosine kinase inhibitors. Int J Chron Obstruct Pulmon Dis. 2014;9:1053-1058.

11. Jones PW, Adamek L, Nadeau G, Banik N. Comparisons of health status scores with MRC grades in COPD: implications for the GOLD 2011 classification. Eur Respir J. 2013;42(3):647-654.

12. Cruz J, Marques A, Jácome C, Gabriel R, Figueiredo D. Global Functioning of COPD Patients With and Without Functional Balance Impairment: An Exploratory Analysis Based on the ICF Framework. COPD. 2015;12(2):207-216.

13. Neder JA, O'Donnell CD, Cory J, et al. Ventilation Distribution Heterogeneity at Rest as a Marker of Exercise Impairment in Mild-toAdvanced COPD. COPD. 2015;12(3):252-259.

14. Putcha N, Drummond MB, Wise RA, Hansel NN. Comorbidities and Chronic Obstructive Pulmonary Disease: Prevalence, Influence on Outcomes, and Management. Semin Respir Crit Care Med. 2015; 36(4):575-591.

15. Mörth C, Valachis A. Single-agent versus combination chemotherapy as first-line treatment for patients with advanced non-small cell lung cancer and performance status 2: a literature-based meta-analysis of randomized studies. Lung Cancer. 2014;84(3):209-214.

16. de Sanctis A, Taillade L, Vignot S, et al. Pulmonary toxicity related to systemic treatment of nonsmall cell lung cancer. Cancer. 2011; 117(14):3069-3080.

17. Beasley MB, Brambilla E, Travis WD. The 2004 World Health Organization classification of lung tumors. Semin Roentgenol. 2005;40(2): 90-97.

18. Global Initiative for Chronic Obstructive Lung Disease [webpage on the Internet]. Global strategy for the diagnosis, management and prevention of COPD, global initiative for Chronic Obstructive Lung Disease (GOLD) 2017. Available from: https://goldcopd.org/gold-2017-global-strategydiagnosis-management-prevention-copd/. Accessed July 17, 2018.

19. Kawai T, Yakumaru K, Suzuki M, Kageyama K. Diffuse interstitial pulmonary fibrosis and lung cancer. Acta Pathol Jpn. 1987;37(1): $11-19$.

20. Watanabe N, Taniguchi H, Kondoh Y, et al. Efficacy of chemotherapy for advanced non-small cell lung cancer with idiopathic pulmonary fibrosis. Respiration. 2013;85(4):326-331.

21. Minegishi Y, Sudoh J, Kuribayasi H, et al. The safety and efficacy of weekly paclitaxel in combination with carboplatin for advanced nonsmall cell lung cancer with idiopathic interstitial pneumonias. Lung Cancer. 2011;71(1):70-74.

22. Mok TS, Wu YL, Thongprasert S, et al. Gefitinib or carboplatinpaclitaxel in pulmonary adenocarcinoma. $N$ Engl J Med. 2009;361(10): 947-957. 
23. Fidias PM, Dakhil SR, Lyss AP, et al. Phase III study of immediate compared with delayed docetaxel after front-line therapy with gemcitabine plus carboplatin in advanced non-small-cell lung cancer. J Clin Oncol. 2009;27(4):591-598.

24. Paz-Ares LG, de Marinis F, Dediu M, et al. PARAMOUNT: Final overall survival results of the phase III study of maintenance pemetrexed versus placebo immediately after induction treatment with pemetrexed plus cisplatin for advanced nonsquamous non-small-cell lung cancer. J Clin Oncol. 2013;31(23):2895-2902.

25. Fossella F, Pereira JR, von Pawel J, et al. Randomized, multinational, phase III study of docetaxel plus platinum combinations versus vinorelbine plus cisplatin for advanced non-small-cell lung cancer: the TAX 326 study group. J Clin Oncol. 2003;21(16):3016-3024.

26. Scagliotti GV, Parikh P, von Pawel J, et al. Phase III study comparing cisplatin plus gemcitabine with cisplatin plus pemetrexed in chemotherapy-naive patients with advanced-stage non-small-cell lung cancer. J Clin Oncol. 2008;26(21):3543-3551.

27. Hirsch FR, Spreafico A, Novello S, Wood MD, Simms L, Papotti M. The prognostic and predictive role of histology in advanced non-small cell lung cancer: a literature review. J Thorac Oncol. 2008;3(12): 1468-1481.

28. Wang M, Li G, Yang Z, et al. Uncoupling protein 2 downregulation by hypoxia through repression of peroxisome proliferator-activated receptor $\gamma$ promotes chemoresistance of non-small cell lung cancer. Oncotarget. 2017;8(5):8083-8094.

29. Lilenbaum R, Villaflor VM, Langer C, et al. Single-agent versus combination chemotherapy in patients with advanced non-small cell lung cancer and a performance status of 2: prognostic factors and treatment selection based on two large randomized clinical trials. J Thorac Oncol. 2009;4(7):869-874.
30. Choi KJ, Cha SI, Shin KM, et al. Prevalence and predictors of pulmonary embolism in Korean patients with exacerbation of chronic obstructive pulmonary disease. Respiration. 2013;85(3):203-209.

31. Wark PA, Tooze M, Powell H, Parsons K. Viral and bacterial infection in acute asthma and chronic obstructive pulmonary disease increases the risk of readmission. Respirology. 2013;18(6):996-1002.

32. Yamanashi K, Marumo S, Shoji T, et al. The relationship between perioperative administration of inhaled corticosteroid and postoperative respiratory complications after pulmonary resection for non-small-cell lung cancer in patients with chronic obstructive pulmonary disease. Gen Thorac Cardiovasc Surg. 2015;63(12):652-659.

33. Takegahara K, Usuda J, Inoue T, Ibi T, Sato A. Preoperative management using inhalation therapy for pulmonary complications in lung cancer patients with chronic obstructive pulmonary disease. Gen Thorac Cardiovasc Surg. 2017;65(7):388-391.

34. Elsayad K, Samhouri L, Scobioala S, Haverkamp U, Eich HT. Is tumor volume reduction during radiotherapy prognostic relevant in patients with stage III non-small cell lung cancer? J Cancer Res Clin Oncol. 2018;144(6):1165-1171.

35. Käsmann L, Niyazi M, Blanck O, et al. Predictive and prognostic value of tumor volume and its changes during radical radiotherapy of stage III non-small cell lung cancer: A systematic review. Strahlenther Onkol. 2018;194(2):79-90.

36. Antonia SJ, Villegas A, Daniel D, et al. Durvalumab after Chemoradiotherapy in Stage III Non-Small-Cell Lung Cancer. $N$ Engl J Med. 2017;377(20):1919-1929.
International Journal of COPD

\section{Publish your work in this journal}

The International Journal of COPD is an international, peer-reviewed journal of therapeutics and pharmacology focusing on concise rapid reporting of clinical studies and reviews in COPD. Special focus is given to the pathophysiological processes underlying the disease, intervention programs, patient focused education, and self management protocols.

\section{Dovepress}

This journal is indexed on PubMed Central, MedLine and CAS. The manuscript management system is completely online and includes a very quick and fair peer-review system, which is all easy to use. Visit http://www.dovepress.com/testimonials.php to read real quotes from published authors. 